Irons M, Sadeghi-Najad A, McCauley R, Wheeler P. Gomez-Lopez-Hernandez syndrome: Expansion of the phenotype. Am I Med Genet October 2000;94:405-408). (Respond: Patricia G Wheeler MD, Division of Genetics, NEMC Box 394, New England Medical Center, 750 Washington St, Boston, MA 02111).

COMMENT. The GLH syndrome is rare, only 8 cases reported. Physical findings that should alert suspicion are a high forehead with turricephaly, hypertelorism, narrowed palpebral fissures, temporal balding, posteriorly rotated ears, long fingers with Dupuytren contracture, and short stature. Corneal opacities may develop over time because of trigeminal anesthesia and corneal insensitivity. Head banging and head rocking, and other self-injurious behavior may occur as a result of pain insensitivity. Psychiatric problems may lead to increased risk of suicide. MRI is preferable to CT in demonstration of the cerebellar anomaly. Rhombencephalosynapsis consists of fusion of the cerebellar hemispheres and absence of the cerebellar vermis. In Greenfield's Neuropathology text (London, Arnold, 1958;p335), aplasia of the cerebellum, due to failure of the two alar plates of the rhombencephalon to unite in the formation of the corpus cerebelli, is called palaeocerebellar aplasia (cerebellar rachischisis). Various nuclear atrophies are also associated, including the inferior olives and dentate nuclei.

\title{
MRI IN MOBIUS SYNDROME
}

The MRI findings in three patients with Mobius syndrome are reported from the Hospital Vall d'Hebron, Barcelona, Spain. An 8 year-old girl had facial diplegia and convergent strabismus secondary to bilateral palsy of the facial and abducens nerves. Associated anomalies included blindness with coloboma, deafness, ear malformation, micrognatia, and mental retardation. MRI showed alterations in the shape of the brainstem and floor of the fourth ventricle, at the level of the inferior pons, with absence of the medial eminence.

A 28 year-old woman presented with congenital bilateral facial palsy, and convergent strabismus with paralysis of horizontal gaze. Associated features included small mandible, deafness, tongue hemiatrophy, and swallowing difficulties. MRI showed brainstem anomalies, including straightening of the floor of the fourth ventricle, absence of the medial colliculus of the pons (VIth and VIIth nuclei), and absence of the hypoglossal eminence in the medulla oblongata (XIIth hypoglossal nuclei). Brainstem auditory evoked potentials were abnormal. Another 28 year-old woman was referred because of headache. She had facial diplegia and convergent strabismus, with limited bilateral ocular abduction. MRI showed a V-shaped depression in the floor of the fourth ventricle due to absence of the facial colliculus. (Pedraza S, Gamez J, Rovira A, Zamora A et al. MRI findings in Mobius syndrome: correlation with clinical features. Neurology October (1 of 2) 2000;55:1058-1060). (Reprints: Dr Salvador Pedraza, Unidad de RM. IDI, Servicio de Radiologia, Hospitals Vall d'Hebron, P. Vall d'Hebron s/n, Barcelona, Spain).

COMMENT. Cranial nerves most often involved in Mobius syndrome are VII, VI, III, and VIII. The IVth, IXth, Xth, and XIIth can also be affected. Anomalies of the cerebellum, hypothalamus, pituitary gland, and limbs are sometimes noted. Genetic, ischemic, infectious, and toxic (thalidomide, alcohol) factors have been suggested in etiology. Congenital agenesis of the brainstem nuclei and malformation of the floor of the fourth ventricle are the most common pathological findings, now demonstrated by MRI. Primary nerve or myopathic involvement has also been suggested in some cases. Electrophysiologic studies support the involvement of brainstem nuclei and internuclear connections. 